\title{
The Seamless Connection of Local and Collective Excited States in Subsystem Time-Dependent Density Functional Theory - Supporting Information -
}

\author{
Johannes Tölle ${ }^{1}$ and Johannes Neugebauer ${ }^{1 *}$ \\ ${ }^{1}$ Theoretische Organische Chemie, Organisch-Chemisches Institut \\ and Center for Multiscale Theory and Computation, \\ Westfälische Wilhelms-Universität Münster \\ Corrensstraße 40, 48149 Münster, Germany
}

Date: $\quad$ January 10, 2022

*email: j.neugebauer@uni-muenster.de 


\section{Computational Details}

All illustrative calculations in this perspective were performed using $\operatorname{SERENITY}^{1,2}$ (V. 1.4) if not stated otherwise. The sTDDFT implementation lacks nuclear gradients but represents otherwise the most complete sTDDFT implementation up to date and is opensource. This includes the capabilities to be used for open-shell systems in an unrestricted formalism ${ }^{3}$ and therefore also allows for the description of triplet-triplet EET.

For the FDEu-TDDFT calculations of the solvated adenine base, the water densities have been initially obtained from isolated calculations using the $\mathrm{BLYP}^{4,5}$ exchange-correlation functional and the def2-TZVP ${ }^{6}$ basis set. All subsystem calculations employed a subsystem only grid associated with the adenine subsystem. The BLYP exchange-correlation and LLP91 ${ }^{7}$ kinetic energy functional were used for non-additive contributions, respectively [LCBLYP $(\mu=0.33) /$ BLYP/LLP91]. In addition, the RI approximation has been applied for the ground- and excited-state Coulomb contributions.

For the PbE-sTDDFT calculations, the LCBLYP functional (for inter- and intra- subsystem contributions) and the def2-SVP ${ }^{6}$ basis set have been used. PbE calculations have been performed using the levelshift projection operator ${ }^{8}$ [LCBLYP/ LCBLYP/level.]. All structures have been optimized using GFN2-XTB ${ }^{9}$ as implemented in the XTB program. ${ }^{10}$ 


\section{References}

[1] Unsleber, J. P.; Dresselhaus, T.; Klahr, K.; Schnieders, D.; Böckers, M.; Barton, D.; Neugebauer, J. Serenity: A subsystem quantum chemistry program. J. Comput. Chem. 2018, 39, 788.

[2] Barton, D.; Bensberg, M.; Böckers, M.; Dresselhaus, T.; Eschenbach, P.; Hellmann, L.; Klahr, K.; Massolle, A.; Niemeyer, N.; Schnieders, D.; Tölle, J.; Unsleber, J. P.; Neugebauer, J. qcserenity/serenity: Release 1.4.0. 2021; DOI: 10.5281/ZENODO.4032651.

[3] Böckers, M.; Neugebauer, J. Excitation Energies of Embedded Open-Shell Systems: Unrestricted Frozen-Density-Embedding Time-Dependent Density-Functional Theory. J. Chem. Phys. 2018, 149, 074102.

[4] Becke, A. D. Phys. Rev. A 1988, 38, 3098-3100.

[5] Lee, C.; Yang, W.; Parr, R. G. Development of the Colle-Salvetti correlation-energy formula into a functional of the electron density. Phys. Rev. B 1988, 37, 785.

[6] Weigend, F.; Ahlrichs, R. Balanced basis sets of split valence, triple zeta valence and quadruple zeta valence quality for $\mathrm{H}$ to Rn: Design and assessment of accuracy. Phys. Chem. Chem. Phys. 2005, 7, 3297-3305.

[7] Lee, H.; Lee, C.; Parr, R. G. Conjoint gradient correction to the Hartree-Fock kineticand exchange-energy density functionals. Phys. Rev. A 1991, 44, 768.

[8] Manby, F. R.; Stella, M.; Goodpaster, J. D.; Miller, III, T. F. A Simple, Exact Density-Functional-Theory Embedding Scheme. J. Chem. Theory Comput. 2012, 8, $2564-2568$.

[9] Bannwarth, C.; Ehlert, S.; Grimme, S. GFN2-xTB-An accurate and broadly parametrized self-consistent tight-binding quantum chemical method with multipole 
electrostatics and density-dependent dispersion contributions. J. Chem. Theory Comput. 2019, 15, 1652-1671.

[10] Bannwarth, C.; Caldeweyher, E.; Ehlert, S.; Hansen, A.; Pracht, P.; Seibert, J.; Spicher, S.; Grimme, S. Extended tight-binding quantum chemistry methods. WIREs Comput. Mol. Sci. 2021, 11, e1493. 